\title{
The clinical features and outcomes of Tolosa-Hunt syndrome
}

\author{
Hyuna Kim ${ }^{1}$ and Shin Yeop Oh${ }^{2 *}$
}

\begin{abstract}
Background: The objective of this study was to investigate the clinical features and outcomes of Tolosa-Hunt syndrome (THS).

Methods: A retrospective review of the medical records was performed on patients with THS between March 2016 and January 2020. A total of eleven patients fulfilling the International Classification of Headache Disorders (ICHD-3 beta) diagnostic criteria for THS were included in this study.

Results: The average age of the patients with THS was $57.18 \pm 15.32$ years and the mean duration of recovery was $26.91 \pm 24.35$ days. All eleven patients had orbital or periorbital pain as the first symptom followed by diplopia. Ptosis was found in five patients (45.45\%) in the involved eye. Sixth cranial nerve (CN) palsy was most common (eight cases, $72.73 \%$ ), followed by third and fourth CN palsy (five cases, $45.45 \%$, respectively), optic neuritis (two cases, $18.18 \%$ ), and trigeminal nerve and facial nerve palsy (one case, $9.09 \%$, respectively). One patient with optic neuritis failed to recover visual acuity and the other ten patients completely recovered their ocular motor limitation. All patients were initially treated with steroids. One patient relapsed after five weeks and one patient had a history of THS five years earlier.

Conclusions: THS responded well to steroid treatment, but if it was accompanied by optic neuritis as optic nerve involvement, we suggest follow-up with high dose steroid treatment especially was important.
\end{abstract}

Keywords: Tolosa-Hunt syndrome, painful ophthalmoplegia, paralysis, orbital pain, optic nerve

\section{Introduction}

Tolosa-Hunt syndrome (THS) is an uncommon idiopathic granulomatous inflammatory disease of the cavernous sinus, superior orbital fissure, or orbit associated with ocular pain and ophthalmoplegia [1, 2]. However, the etiology of THS is still unknown. Thus, the diagnosis of THS should exclude other causes of painful ophthalmoplegia such as tumors, vasculitis, meningitis, sarcoidosis, diabetic ophthalmoplegia, and pseudotumor $[3,4]$. THS is described in the International Classification of Headache Disorders (ICHD 3-beta) diagnostic criteria as

\footnotetext{
* Correspondence: mojili914@hanmail.net

${ }^{2}$ Department of Ophthalmology, Samsung Changwon Hospital, Sungkyunkwan University School of Medicine, 158, Paryong-ro, Masanhoewon-gu, 51353 Changwon, Korea

Full list of author information is available at the end of the article
}

unilateral orbital pain associated with paresis of one or more of the third, fourth, and/or sixth cranial nerves caused by a granulomatous inflammation in the cavernous sinus, superior orbital fissure or orbit (Table 1) [5]. In clinical practice, the disorder is diagnosed based on the above diagnostic criteria and treatment with corticosteroids is generally recommended. There are relatively few reports on the clinical course of THS, so the objective of this retrospective study was to investigate the clinical outcomes of THS.

\section{Methods}

The Institutional Review Board for Human studies at Samsung Changwon Hospital (Changwon, Republic of Korea) reviewed and approved this study protocol (IRB No. 2020-06-014). As this study was a retrospective 
Table 1 ICHD-3 beta diagnostic criteria for 13.7 Tolosa-Hunt syndrome

\begin{tabular}{l} 
A. Unilateral headache fulfilling criterion C \\
\hline B. Both of the following: \\
1. Granulation inflammation of the cavernous sinus, superior orbital \\
fissure or orbit, demonstrated by MRI or biopsy \\
2. Paresis of one or more of the ipsilateral third, fourth and/or sixth \\
cranial nerves \\
C. Evidence of causation demonstrated by both of the following: \\
1. Headache has preceded paresis of the third, fourth and/or sixth \\
cranial nerves by 2 weeks, or developed with it \\
2. Headache is localized around the ipsilateral brow and eye \\
D. Not better accounted for by another ICHD-3 diagnosis.
\end{tabular}

ICHD 3 beta $=$ International Classification of Headache Disorders, third edition $\mathrm{MRI}=$ magnetic resonance imaging

study, informed consent was obtained from all patients before the imaging examination and all identifiable personal information of patients would be anonymized. All study conduct adhered to the tenets of the Declaration of Helsinki for the use of human participants in biomedical research. The medical records of patients diagnosed with THS from March 2016 to January 2020 at Samsung Changwon Hospital were retrospectively reviewed. The diagnosis of THS was defined by (1) unilateral headache or orbital pain; (2) headache preceded with paresis of the third cranial nerve $(\mathrm{CN})$, fourth $\mathrm{CN}$, and/or sixth $\mathrm{CN}$ for two weeks, or developed with it; and (3) headache localized around the ipsilateral brow and eye based on ICHD-3 beta criteria [5]. All patients underwent brain or orbit magnetic resonance imaging (MRI) and enhancing lesions of the cavernous sinus, superior orbital fissure, and/or orbital apex were identified. Furthermore, other causes of painful ophthalmoplegia including tumors, vasculitis, basal meningitis, sarcoidosis, migraine, cranial herpes zoster, giant cell arteritis, myositis, pseudotumor, and thyroid ophthalmopathy were excluded. Patients who did not show confirmed recovery within a follow-up duration of less than six months were excluded. Case notes were reviewed to obtain data on patient demographics (age, sex), previous medical history (hypertension, diabetes mellitus, or cancer), symptoms (recorded as orbital pain, diplopia, ptosis, visual loss, and facial numbness), the involved cranial nerves, laboratory results, neuroimaging, the symptom-resolution interval, and treatment. All patients were given oral methylprednisolone (MPD) at $50 \mathrm{mg} /$ day or intravenous (IV) MPD $1 \mathrm{~g} /$ day initially and then switched to a gradually decreased dose until the signs and symptoms resolved. The patients with previously diagnosed paralytic or restrictive strabismus, congenital strabismus, or with previous extraocular muscle surgery were excluded. At the first visit, the patients underwent ophthalmologic assessments including slit-lamp examination, intraocular pressure measurements, ocular motor limitation assessment, and fundus photography. In addition, the patients were examined the hematological tests and cerebrospinal studies. At the final follow-up visit, THS recovery was deemed complete or non-recovered based on ocular motor limitation and visual acuity. Complete recovery was defined as the absence of ocular motor limitation and other $\mathrm{CN}$ abnormalities and non-recovery was defined as sequelae of $\mathrm{CN}$ function.

\section{Results}

A total of eleven patients (night males and two females) were included in this study and the mean follow-up time was $201.27 \pm 31.36$ days after the initial visit. The average age at THS onset was $57.18 \pm 15.32$ years (range, 35-78 years). All eleven patients had orbital or periorbital pain as the first symptom followed by diplopia or ptosis. Diplopia and ptosis were found in eleven patients (100\%) and five patients (45.45\%), respectively. Sixth $\mathrm{CN}$ palsy was most common (eight cases, $72.73 \%$ ), followed by fourth and sixth $\mathrm{CN}$ palsy (five cases, $45.45 \%$, respectively), optic nerve (ON) involvement (two cases, $18.18 \%$ ), and trigeminal nerve and facial nerve palsy (one case, $9.09 \%$, respectively). At the initial examination, four patients had one $\mathrm{CN}$ involved, three patients had two CNs involved, and two patients had three CNs involved. Complete recovery was seen in ten $(90.91 \%)$ patients and in one patient, the ocular motor limitation was completely recovered but visual acuity was not recovered. The mean duration of recovery was $26.91 \pm$ 24.35 days (range, 8-73 days) (Table 2). The clinical summary and MRI findings of the patients are presented in Table 3. All patients were initially treated with steroid. Six patients received oral MPD at $50 \mathrm{mg} /$ day and five patients received IV MPD at $1 \mathrm{~g} /$ day. The laboratory test results showed an elevated erythrocyte sedimentation rate (ESR) in three patients and no patients had elevated C-reactive protein (CRP) values. Three patients with elevated ESR had a paralysis of all third, fourth and sixth CNs. In two patients with optic neuritis as ON involvement, one had fourth $\mathrm{CN}$ palsy at the first visit with a history of facial nerve palsy before four months. ON swelling was observed three days later, and IV MPD at $1 \mathrm{~g} /$ day was administered. Another patient had third, fourth, and sixth $\mathrm{CN}$ palsy at the first visit and oral $\mathrm{MPD}$ at $50 \mathrm{mg} /$ day was administered. After five weeks, decreased visual acuity was observed and IV MPD at $1 \mathrm{~g} /$ day was injected. The first patient recovered completely after treatment, but the second patient did not recover from visual impairment. Of all the patients, two patients relapsed, and one patient had recurrence of visual impairment at 5 weeks interval. The other patient had a history of treatment for THS five years prior according to the medical record and was included as a relapsed patient. 
Table 2 Demographics of patients with Tolosa-Hunt syndrome

\begin{tabular}{|c|c|}
\hline Parameters & \\
\hline Total patients (n) & 11 \\
\hline Male : Female (n) & $9(81.12 \%): 2(18.18 \%)$ \\
\hline Age at time of onset (years) & $57.18 \pm 15.32$ (range : $35-78)$ \\
\hline Involved eye (right : left) & 5 (45.45 \%) : 6 (54.55 \%) \\
\hline Duration of follow-up (days) & $201.27 \pm 31.36$ \\
\hline Duration of recovery (days) & $26.91 \pm 24.35$ (range : 8-73) \\
\hline Complete recovery state (n) & $10(90.91 \%)$ \\
\hline ESR (mm/hour) & $16.73 \pm 13.76$ (range : 2-52) \\
\hline CRP (mg/L) & $1.15 \pm 0.84$ (range : 0.3-2.6) \\
\hline Diplopia (n) & $11(100 \%)$ \\
\hline Periorbital pain (n) & $11(100 \%)$ \\
\hline $\begin{array}{l}\text { CN nerve involvement cases ( } \mathrm{n} \text { ) } \\
\text { Optic nerve } \\
\text { Third CN } \\
\text { Fourth CN } \\
\text { Trigeminal nerve } \\
\text { Sixth CN }\end{array}$ & $\begin{array}{l}2(18.18 \%) \\
5(45.45 \%) \\
5(45.45 \%) \\
1(9.09 \%) \\
8(72.73 \%)\end{array}$ \\
\hline Ptosis (n) & $5(45.45 \%)$ \\
\hline Hypertension (n) & $3(27.27 \%)$ \\
\hline Diabetes mellitus ( $\mathrm{n}$ ) & $2(18.18 \%)$ \\
\hline Recurrence state (n) & $2(28.18 \%)$ \\
\hline
\end{tabular}

Values are presented as mean \pm SD

$\mathrm{SD}=$ standard deviation

$\mathrm{ESR}=$ erythrocyte sedimentation rate; $\mathrm{CRP}=\mathrm{C}$-reactive protein $\mathrm{CN}=$ cranial nerve

\section{Discussion}

This retrospective study of eleven cases of THS based on ICHD-3 beta criteria showed that the clinical manifestations were variable. The sixth $\mathrm{CN}(72.73 \%)$ was most commonly involved, followed by the third (45.45\%), and fourth CN (45.45\%). Previous studies reported a high rate of third $\mathrm{CN}$ (78-91\%) involvement $[6,7]$, however, Curone et al. reported a low rate of third $\mathrm{CN}$ (39\%) involvement [8]. As such, $\mathrm{CN}$ involvement has been variably reported in each study. THS is usually treated with steroid, but there are no rules regarding drugs, formulations, routes of administration, or therapeutic doses. Steroid treatment generally includes prednisone, MPD, and dexamethasone given orally or by IV injection. In this study, five patients were treated with IV MPD at $1 \mathrm{~g} /$ day for three days, and six patients received oral MPD at $50 \mathrm{mg} /$ day for seven days, then the dosage was tapered. The difference in treatment dose was determined in consideration of the patient's age, the severity of their symptoms, and underlying diseases. After the initiation of MPD treatment, pain resolved within two days in all patients, and ocular motor limitation gradually improved. Other studies reported that most patients achieved pain relief within 72 hours and that $\mathrm{CN}$ palsy resolved over two to eight weeks $[6,7]$. In the patients in this study, the recovery period varied from one to eight weeks, similar to the previous report. Steroid treatment relieves the pain quickly, but the signs do not resolve immediately after treatment. As in this study and previous studies, because steroid administration had dramatic effects, when THS is diagnosed, it is important to start steroid therapy immediately. An accurate diagnosis is important for quick and appropriate treatment, but many diseases must be considered in the differential diagnosis of painful ophthalmoplegia. Tolosa-Hunt syndrome is a diagnosis of exclusion requiring various examination and careful evaluation to rule out tumors, vascular causes, or other forms of inflammation in the lesion of the cavernous sinus and superior orbital fissure (Table 4). The role of MRI in the criteria has been included as "granulomatous inflammation demonstrated by MRI or biopsy" in the ICHD-3 beta criteria. In most studies and our study, no patient had a biopsy or pathological examination $[3,4,6,7]$. Also, the location of lesion is an important factor in the differential diagnosis, especially an orbital pseudotumor with characteristics and steroid response similar to THS [9]. Therefore, MRI plays a key role in the diagnosis of THS. Since THS can be diagnosed by anatomical location, MRI should be performed if THS is suspected in a case of painful ophthalmoplegia. The recurrence rate in our study (18.18\%) was similar to that of Rui et al. (23\%) [6] and Colnaghi et al. (21\%) [3] but was lower than that reported by Zhang et al. (37\%) [7]. Two patients in this study experienced recurrences, one recurred after five weeks and the other had a history of THS diagnosis five years earlier. THS patients have a good prognosis but recurrences occur in about $21-50 \%$ of the cases over an interval of months to years $[3,7,10]$. Thus, it is important to consider follow-up and the possibility of a recurrence after a full recovery. In this study, the laboratory test results showed elevated ESR values in three patients, which were the patients with all third, fourth, and sixth $\mathrm{CN}$ involvement. However, no patients with non-elevated ESR had paralysis of all third, fourth, and sixth CNs. In patients with elevated ESR, one patient did not recover from visual impairment, and the other two showed complete recovery, but the recovery period of the three patients was about 2 months, which was longer than the average recovery period of all patients. The longer recovery period can be considered in association with the clinical findings that there are many involved $\mathrm{CN}$ palsy, and the ESR values could be considered as a factor predicting the severity of the disease and the recovery period. However, it is expected that further studies of the blood test results in more patients will be needed in 
Table 3 Clinical summary of patients with Tolosa-Hunt Syndrome

\begin{tabular}{|c|c|c|c|c|c|c|c|c|c|}
\hline Case & Sex & Age & Clinical findings & Involved CN & MRI findings & & $\begin{array}{l}\text { CRP } \\
(0-5)\end{array}$ & $\begin{array}{l}\text { Treatment } \\
\text { (per day) }\end{array}$ & Relapse \\
\hline 1 & $M 7$ & 73 & Diplopia & $6 \mathrm{CN}$ & $\begin{array}{l}\text { Enhanced left cavernous sinus and } \\
\text { clinoid process }\end{array}$ & 15 & 0.9 & Oral MPD $50 \mathrm{mg}$ & - \\
\hline 2 & $M 7$ & 74 & Diplopia, Ptosis & Optic nerve, 3/4/6 CN & $\begin{array}{l}\text { Enlarged and enhanced left } \\
\text { cavernous sinus/apex/optic nerve }\end{array}$ & 52 & 2.6 & Oral MPD 50 mg & 5 weeks interval \\
\hline 3 & $M 5$ & 52 & Diplopia & Optic nerve, 4 CN & $\begin{array}{l}\text { Mild thickening and enhanced right } \\
\text { cavernous sinus/apex (superior/ } \\
\text { inferior orbital fissure) }\end{array}$ & 10 & 2.5 & IV MPD $1 \mathrm{~g}$ & - \\
\hline 4 & F 4 & 46 & Diplopia, Ptosis & $3 / 4 / 6 \mathrm{CN}$ & $\begin{array}{l}\text { Enhanced focal triangular shaped } \\
\text { right cavernous sinus with enlarged } \\
\text { right superior ophthalmic vein }\end{array}$ & 21 & 0.3 & IV MPD $1 \mathrm{~g}$ & - \\
\hline 5 & $M 6$ & 68 & Diplopia, Ptosis & $3 \mathrm{CN}$ & $\begin{array}{l}\text { Enhanced focal right superior } \\
\text { orbital fissure }\end{array}$ & 12 & 2.0 & Oral MPD 50 mg & 5 years ago \\
\hline 6 & $M 4$ & 46 & Diplopia & $6 \mathrm{CN}$ & $\begin{array}{l}\text { Slightly bulging of right cavernous } \\
\text { sinus }\end{array}$ & 2 & 0.5 & Oral MPD 50 mg & - \\
\hline 7 & M 4 & 46 & Diplopia & $6 \mathrm{CN}$, facial nerve & $\begin{array}{l}\text { Suspicious enhanced left superior } \\
\text { orbital fissure }\end{array}$ & 10 & 0.7 & Oral MPD $50 \mathrm{mg}$ & - \\
\hline 8 & $M 6$ & 69 & Diplopia & $4 \mathrm{CN}$ & $\begin{array}{l}\text { Enhanced left cavernous sinus/ } \\
\text { superior orbital fissure }\end{array}$ & 8 & 0.8 & Oral MPD 50 mg & - \\
\hline 9 & $M 7$ & 78 & Diplopia, Ptosis & $3 / 6 \mathrm{CN}$ & $\begin{array}{l}\text { Enlarged and enhanced left } \\
\text { cavernous sinus }\end{array}$ & 11 & 0.7 & IV MPD $1 \mathrm{~g}$ & - \\
\hline 10 & F 4 & 42 & Diplopia, Ptosis & $3 / 4 / 6 \mathrm{CN}$ & $\begin{array}{l}\text { Enhanced right cavernous sinus } \\
\text { and extending to orbital apex }\end{array}$ & 30 & 0.5 & IV MPD $1 \mathrm{~g}$ & - \\
\hline 11 & $M 3$ & 35 & Diplopia & Trigeminal nerve, $6 \mathrm{CN}$ & $\begin{array}{l}\text { Enhanced left cavernous sinus and } \\
\text { extracranial portion of mandibular } \\
\text { division of trigeminal nerve }\end{array}$ & 13 & 1.1 & IV MPD $1 \mathrm{~g}$ & - \\
\hline
\end{tabular}

$\mathrm{MRI}=$ magnetic resonance imaging; $\mathrm{ESR}=$ erythrocyte sedimentation rate; $\mathrm{CRP}=\mathrm{C}$-reactive protein

$\mathrm{CN}=$ cranial nerve; $\mathrm{MPD}=$ methylprednisolone; IV = intravenous

the future. Meanwhile, ON dysfunction has been reported, indicating that the pathological process may involve the orbital apex [10]. Two patients in this study had optic neuritis as ON involvement and enhanced apex lesions were confirmed by orbit MRI. One patient had fourth $\mathrm{CN}$ palsy and optic neuritis and recovered completely after steroid treatment. In contrast, in other patients with third, fourth, and sixth CN palsy, relapsed five weeks later with retrobulbar optic neuritis and third,

Table 4 Diagnostic evaluation of Tolosa-Hunt syndrome

A. Laboratory tests, Complete blood count, Serum chemistry (electrolyte, glucose, liver and renal function), Erythrocyte sedimentation rate (ESR), C-reactive protein (CPR), Hemoglobin A1C (HbA1C), Angiotensin converting enzyme (ACE), Thyroid function test, Auto-antibodies

B. Cerebrospinal fluid (CSF) studies

C. Neuro-radiological studies, Brain or Orbit MRI, Orbit CT, Cerebral angiography

D. Ophthalmological examinations, Visual acuity, Intraocular pressure $(\mathrm{IOP})$, Slit lamp examination, Fundus examination, Extraocular muscle movement

E. Biopsy of cavernous sinus (in some cases)

$\mathrm{MRI}=$ magnetic resonance imaging; $\mathrm{CT}=$ computed tomography fourth, and sixth CN palsy. After high-dose steroid treatment, the ocular motor limitation was completely recovered, but the vision loss (recognition of hand motion) did not improve and a pale optic disc was observed at follow-up. Kline and Hoyt [10] reported that the optic disc may be normal, swollen, or pale in appearance, with visual decline ranging from minimal to blindness, and variable final visual acuity. In the two cases in this study, the opposite results were found. In one case, the visual acuity was completely recovered and in the other case, it was not recovered despite the same treatment. In patients whose vision did not recover, oral MPD at $50 \mathrm{mg} /$ day was prescribed at the initial examination because of old age and uncontrolled diabetic history. At recurrence of THS with optic neuritis, $1 \mathrm{~g}$ of MPD was administered, but the loss of vision did not recover. It is thought that the damaged $\mathrm{ON}$ axons could not be recovered due to persistent granulomatous inflammation. We suggest that initial IV MPD at $1 \mathrm{~g} /$ day might have a better prognosis considering the elevated ESR and extended inflammation of cavernous sinus and orbital apex. Although optic neuritis as ON involvement is not common in THS, the occurrence of ON dysfunction should be confirmed through a visual acuity examination during the 
follow-up period and immediate high dose steroid treatment should be initiated. In addition, even if the ocular motor limitation have improved, it is necessary to check for recurrence or $\mathrm{ON}$ function through sufficient followup. Our study had several limitations. First, it was a retrospective, single-hospital-based study, suggesting possible selection bias. Second, the sample size was small due to the relatively uncommon occurrence of the disease. Third, the follow-up intervals were not standardized and were not long-term. Nevertheless, this study showed the strength of analyzing eleven cases and reporting various THS clinical symptoms. In conclusion, considering that THS may cause optic neuritis as $\mathrm{ON}$ involvement, immediate high dose steroid treatment should be performed at the time of diagnosis. In addition, since there is a possibility of recurrence, longterm follow-up is necessary.

\section{Acknowledgements}

Not applicable.

\section{Authors' contributions}

Conception or design of the work (Shin Yeop Oh); analysis and interpretation (Hyuna Kim, Shin Yeop Oh); data collection and drafting the work (Shin Yeop Oh); critical revision of the article (Hyuna Kim, Shin Yeop Oh); final approval of the version (Shin Yeop Oh).

\section{Funding}

This work was supported by the National Research Foundation of Korea [NRF-2020R1F1A1072382]. The funder had no role in study design, data collection and analysis, decision to publish, or preparation of the manuscript.

\section{Availability of data and materials}

The dataset used and/or analysed during the current study are available from the corresponding author on reasonable request.

\section{Declarations}

\section{Ethics approval and consent to participate}

The IRB for Human studies at Samsung Changwon Hospital (Changwon, Republic of Korea) reviewed and approved this study protocol (IRB No. 202006-014). Informed consent was obtained from all patients (retrospective study)

\section{Consent for publication}

Not applicable.

\section{Competing interests}

The author declare that they have no competing interests.

\section{Author details \\ 'Department of Ophthalmology, Soonchunhyang University Seoul Hospital, Soonchunhyang University College of Medicine, Seoul, Korea. ${ }^{2}$ Department of Ophthalmology, Samsung Changwon Hospital, Sungkyunkwan University School of Medicine, 158, Paryong-ro, Masanhoewon-gu, 51353 Changwon, Korea.}

Received: 28 January 2021 Accepted: 20 May 2021

Published online: 27 May 2021

\section{References}

1. Tolosa E. Periarteritic lesions of the carotid siphon with the clinical features of a carotid infraclinoidal aneurysm. J Neurol Neurosurg Psychiatry. 1954;17: $300-2$.
2. Hunt WE, Meagher JN, LeFever HE, Zeman W. Painful opthalmoplegia. Its relation to indolent inflammation of the carvernous sinus. Neurology. 1961; 11:56-62.

3. Colnaghi S, Versino M, Marchioni E, Pichiecchio A, Bastianello S, Cosi V, et al. ICHD-II diagnostic criteria for Tolosa-Hunt syndrome in idiopathic inflammatory syndromes of the orbit and/or the cavernous sinus. Cephalagia. 2008;28:577-84.

4. LaMantia L, Curone M, Rapaport AM, Bussone G. Tolosa-Hunt syndrome: critical literature review based on HIS 2004 criteria. Cephalagia. 2006;26:77281.

5. Classification Committee of the International Headache Society. The international classification of headache disorders, 3rd edition (beta version). Cephalagia. 2013;33:629-808.

6. Hao R, He Y, Zhang H, Zhang W, Li X, Ke Y. The evaluation of ICHD-3 beta diagnostic criteria for Tolosa-Hunt syndrome: a study of 22 cases of TolosaHunt syndrome. Neurol Sci. 2015;36:899-905.

7. Zhang X, Zhou Z, Steiner TJ, Zhang W, Liu R, Dong Z, et al. Validation of ICHD-3 beta diagnostic criteria for 13.7 Tolosa-Hunt syndrome: Analysis of 77 cases of painful ophthalmoplegia. Cephalagia. 2014;34:624-32.

8. Curone M, Tullo V, Cecchini AP, Peccarisi C, Leone M, Bussone G. Painful ophthalmoplegia: a retrospective study of 23 cases. Neurol Sci. 2009;30:133-

9. Wasmeier C, Pfadenhauer K, Rosler A. Idiopathic inflammatory pseudotumor of the orbit and Tolosa-Hunt syndrome-are they the same disease? J Neurol. 2002;249:1237-41.

10. Kline LB, Hoyt WF. The Tolosa-Hunt syndrome. J Neurol Neurosurg Psychiatry. 2001; 71:577-82.

\section{Publisher's Note}

Springer Nature remains neutral with regard to jurisdictional claims in published maps and institutional affiliations.
Ready to submit your research? Choose BMC and benefit from:
- fast, convenient online submission
- thorough peer review by experienced researchers in your field
- rapid publication on acceptance
- support for research data, including large and complex data types
- gold Open Access which fosters wider collaboration and increased citations
- maximum visibility for your research: over $100 \mathrm{M}$ website views per year
At BMC, research is always in progress.
Learn more biomedcentral.com/submissions 